\title{
SINOPSIS DE IPOMOEA L. Y MERREMIA DENNST. EX ENDL. (CONVOLVULACEAE) EN EL NORTE DEL PERÚ (LAMBAYEQUE Y TERRITORIOS ADYACENTES)
}

\author{
Luciana ALARCÓN-BRAVO ${ }^{1,2^{*}}$, Gabriela TORRES-REAÑO ${ }^{1,2^{*}}$, Daniel F. AUSTIN ${ }^{3 \dagger}$, \\ Consuelo ROJAS-IDROGO ${ }^{1} \&$ Guillermo E. DELGADO-PAREDES ${ }^{1}$ \\ ${ }^{1}$ Facultad de Ciencias Biológicas, Universidad Nacional Pedro Ruiz Gallo, Ciudad Universitaria, \\ Juan XXIII 391, Lambayeque, Perú. \\ ${ }^{2}$ Asociación Lambayecana de Botánicos (ASLAB), Chiclayo, Perú. \\ ${ }^{3}$ Conservation and Science Department, Sonora Desert Museum, $2021 \mathrm{~N}$ \\ Kinney Road, Tucson, Arizona 85743.
}

*Autores para correspondencia: luanalbr13901@gmail.com, anag521986@gmail.com

Recibido el 2 de noviembre de 2016, aceptado para su publicación el 21 de noviembre de 2016

RESUMEN. Sinopsis de Ipomoea L. y Merremia Dennst. Ex Endl. (Convolvulaceae) en el Norte del Perú (Lambayeque y territorios adyacentes). El objetivo principal de este trabajo es identificar y comentar los registros anteriores y nuevos de los géneros Ipomoea y Merremia en el departamento de Lambayeque (Perú) y zonas circundantes. Se describen 18 especies de Ipomoea y 3 especies de Merremia: Ipomoea alba, I. asarifolia, I. hederifolia, I. indica, I. nil e I. wrightii. I. aristolochiifolia, I. batatas, I. cairica, I. carnea, I. incarnata, I. parasitica, I. piurensis, I. purpurea, I. tiliacea, Merremia aegyptia, M. quinquefolia y M. umbellata son nuevos registros para la flora de Lambayeque. Incluso Ipomoea amnicola, I. dumetorum e I. quamoclit se reportan como nuevos registros en el departamento adyacente de Cajamarca. Se presentan claves para identificar las especies y los grupos intergenéricos. La distribución de cada especie es también presentada para mostrar su biogeografía.

Palabras clave. Ipomoea, Merremia, Convolvulaceae, taxonomía, distribución, Lambayeque, Perú.

\begin{abstract}
Synopsis of Ipomoea L. and Merremia Dennst. Ex Endl. (Convolvulaceae) in the North of Peru (Lambayeque and surrounding areas). The main of this paper is to identifie and to comment previous and new records of Ipomoea and Merremia in Lambayeque Department (Peru) and surrounding areas. 18 species of Ipomoea and 3 species of Merremia are reported: Ipomoea alba, I. asarifolia, I. hederifolia, I. indica, I. nil e I. wrightii. I. aristolochiifolia, I. batatas, I. cairica, I. carnea, I. incarnata, I. tiliacea, I. parasitica, I. piurensis, I. purpurea, Merremia aegyptia, M. quinquefolia y M. umbellata are new records for the Lambayeque flora. Even, I. amnicola, I. dumetorum and I. quamoclit were found as new records in the adjacent Cajamarca Department. Keys are provided for to identifie species and infrageneric groups. Distribution of each species is also presented in order to show their biogeography.
\end{abstract}

Key words. Ipomoea, Merremia, Convolvulaceae, taxonomy, distribution, Lambayeque, Perú. 


\section{INTRODUCCIÓN}

La familia Convolvulaceae, ubicada en el corazón de las Euastéridas (APG IV), actualmente comprende 9 tribus, entre las que se encuentran Ipomoeae, con el género Ipomoea dividido en los subgéneros Ipomoea, Eriospermum y Quamoclit (Austin y Huamán, 1996), y Merremieae con el género Merremia (Chiarini \& Ariza, 2006; Felger et al., 2012). Ipomoea se caracteriza por presentar plantas generalmente trepadoras o postradas; estilo íntegro, estigma entero o bilobulado; corola 3-5 cm de largo, blanca o rosácea; anteras no torcidas después de la antesis; polen con exina espinosa, porado; cápsula dehiscente por 4 valvas, generalmente con 4 semillas glabras, pilosas o lanuginosas en las costillas longitudinales, y las especies del subgénero Ipomoea son las que presentan tubos más claros que el limbo, de 4 a $8.5 \mathrm{~cm}$; estigmas trilobulados; sépalos herbáceos; pedúnculos y pedicelos con tricomas reflexos o erectos; peciólos pubescentes, de $1 \mathrm{a} 14 \mathrm{~cm}$ de longitud; Eriospermum las que tienen corola infundibuliforme o campanulada, menos hipocrateriforme ni tubular; tubos cortos a largos, violetas, rosados o lilas, algunas veces azules o blancos de 1.8 a 10 cm; estigma 2-3-lobulados y Quamoclit las que tienen corola infundibuliforme, campanulada, hipocrateriforme o tubular, de 1 a $12 \mathrm{~cm}$ de longitud; sépalos glabros o ligeramente puberulentos; estigmas bilobulados o ligeramente bilobulados. Merremia, sin embargo, se diferencia de Ipomoea por la torsión de las anteras durante la antesis. Las características posición y forma de los sépalos persistentes y acrescentes en los frutos de Merremia pueden ser utilizadas fácilmente para diferenciar especies, debido a que comúnmente los sépalos son paralelos al eje longitudinal del fruto, pero en pocos casos llegan a ser perpendiculares como en $M$. cisoides, $M$. dissecta y M. macrocalyx.

En el Perú la familia Convolvulaceae comprende 18 géneros y 151 especies, mayormente bejucos y lianas, donde sólo 21 especies son endémicas (Brako \& Zarucchi, 1993; Ulloa Ulloa et al., 2004). De los géneros Evolvulus, Ipomoea, Jacquemontia y Merremia, 9 especies y una subespecie se reconocen como endemismos peruanos ocupando principalmente la provincia Pacífica, sector Sechurense y la provincia ParamunoPeruana (Llatas et al., 1997). El género Ipomoea comprende aproximadamente 52 especies, de las cuales 4 son endémicas (Austin \& Huamán, 1996; León, 2006), para Lambayeque Brako \& Zarucchi (1993) reportaron 8 especies. El género Merremia comprende aproximadamente 8 especies, de las cuales 2 son endémicas (Mostacero et al., 2002; León, 2006)

Dentro del género Ipomoea encontramos I. batatas e I. aquatica de gran importancia económica y alimenticia (Naskar, 1990; Namanda et al., 2015) e I. purga de interés medicinal (Carranza, 2007; Felger et al., 2012) debido a sus glucoresinas (Wagner, 1973). Asimismo, la familia Convolvulaceae en el norte del Perú se ha estudiado escasamente, pues solo se han reportado algunas especies de Ipomoea y Merremia (Abad, 1983; Llatas, 2011)

El objetivo fundamental del presente trabajo fue determinar y ubicar en sus grupos intergenéricos a las especies de los géneros Ipomoea y Merremia (Convolvulaceae) en el departamento de Lambayeque y zonas aledañas, y elaborar claves taxonómicas para su identificación.

\section{MATERIAL Y MÉTODOS}

La investigación se realizó en el departamento de Lambayeque (5'29'37' -

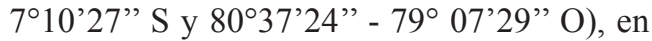
el noroeste del Perú, abarcando también las zonas fronterizas de los departamentos de Piura y Cajamarca. Las exploraciones botánicas se 
realizaron desde las altitudes más bajas (0-200 m) que comprende desde el nivel de las playas a los "algarrobales" de Prosopis, propios del Bosque Estacionalmente Seco, hasta la elevación más alta $(4060 \mathrm{~m})$ que corresponde a la Laguna Minas, al noreste de IncahuasiFerreñafe (Lambayeque), en los bosques montanos de neblina y punas húmedas ("jalca") (Llatas \& López, 2005). Desde el punto de vista biogeográfico, las playas y algarrobales corresponden a la provincia Pacífica (Llatas et al., 1997), y los bosques montanos y la jalca, a la provincia Guayaquileña-Ecuatoriana (Galán de Mera et al., 2015), de acuerdo con la síntesis de Rivas-Martínez et al. (2011) sobre América del Sur.

La recopilación de información, basada en revisiones bibliográficas, publicaciones científicas, colecciones físicas, catálogos virtuales de la base de datos online de Neotropical Herbarium Specimens (The Field Museum), Trópicos (Missouri Botanical Garden) y Herbarium Catalogue (Kew Royal Botanic Gardens) y la base de datos del herbario PRG (Universidad Nacional Pedro Ruiz Gallo), correspondiente a la temática en estudio, determinaron la realización de 18 rutas de exploraciones botánicas a las zonas en estudio en diferentes épocas de los años 2013 y 2014, recolectándose las muestras y los datos más relevantes de cada especie.

La taxonomía de las especies se muestra de acuerdo a la clasificación de Austin (1975a, 1975b, 1979, 1997) y Austin \& Huáman (1996), ubicándose a las especies a nivel infragénerico debido a que las categorías superiores a género no están presentes en las investigaciones más recientes.

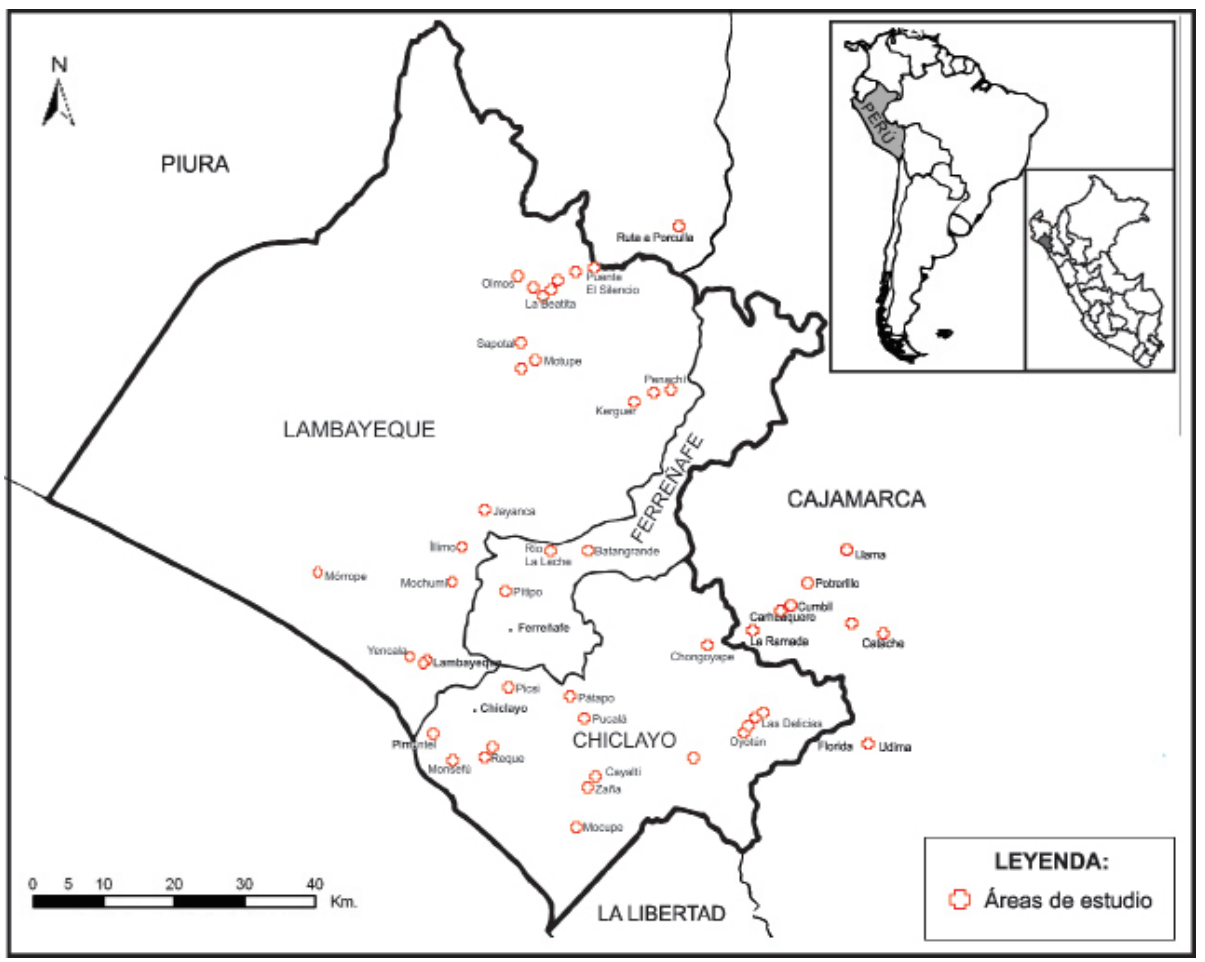

Figura 1. Distribución de los taxones de Ipomoea y Merremia colectados en el departamento de Lambayeque y zonas aledañas (Perú) 


\section{RESULTADOS Y DISCUSIÓN}

Se registraron 18 taxones de Ipomoea pertenecientes a 3 subgéneros y 3 taxones de Merremia, entre ellas se reportan para Lambayeque por primera vez a 12 especies: I. aristolochiifolia, I. batatas, I. cairica, I. carnea, I. incarnata, I. parasitica, I. piurensis, I. purpurea, I. tiliacea, Merremia aegyptia, M. quinquefolia y M. umbellata; para la zona aledaña perteneciente al departamento de Cajamarca se reportan a I. purpurea, I. dumetorum, I. parasitica, I. aristolochiifolia, I. tiliacea e I. quamoclit; para la zona aledaña perteneciente al departamento de Piura se reporta a I. aristolochiifolia.

De la observación y descripción de todos los taxones, la figura 1 muestra el área de distribución de las especies estudiadas y las figuras $2 \mathrm{a}$ y $2 \mathrm{~b}$ las flores de los taxones reportados de los géneros Ipomoea y Merremia.

La distribución en América se abrevia por países de acuerdo a los topónimos siguientes: $\mathrm{EEUU}=$ Estados Unidos, $\mathrm{ME}=$ México, $\mathrm{BH}=$ Bahamas, $\mathrm{BE}=$ Belice, $\mathrm{CR}=$ Costa Rica, $\mathrm{CU}=$ Cuba, DM= Dominica, ES= El Salvador, GT= Guatemala, $\mathrm{HA}=$ Haití, $\mathrm{HO}=$ Honduras, $\mathrm{JA}=$ Jamaica, $\mathrm{NI}=$ Nicaragua, $\mathrm{PN}=$ Panamá, $\mathrm{PR}=$ Puerto Rico, DO= República Dominicana, $\mathrm{AR}=$ Argentina, $\mathrm{BO}=$ Bolivia, $\mathrm{BR}=\mathrm{Brasil}$, $\mathrm{CH}=$ Chile, $\mathrm{CO}=$ Colombia, $\mathrm{EC}=$ Ecuador, $\mathrm{GU}=$ Guyana, $\mathrm{GUF}=$ Guayana Francesa, $\mathrm{PAR}=$ Paraguay, $\mathrm{PE}=$ Perú, $\mathrm{SUR}=$ Surinam, $\mathrm{UR}=$ Uruguay, $\mathrm{VE}=$ Venezuela, $\mathrm{AN}=$ Las Antillas.

La distribución en el Perú se abrevia por departamentos de acuerdo a los topónimos de Brako \& Zarucchi (1993): AM= Amazonas, $\mathrm{AN}=$ Ancash, $\mathrm{AP}=$ Apurímac, $\mathrm{AR}=$ Arequipa, $\mathrm{AY}=$ Ayacucho, $\mathrm{CA}=$ Cajamarca, $\mathrm{CU}=\mathrm{Cuzco}$, $\mathrm{HU}=$ Huánuco, $\mathrm{HV}=$ Huancavelica, $\mathrm{IC}=\mathrm{Ica}$, $\mathrm{JU}=$ Junín, $\mathrm{LA}=$ Lambayeque, $\mathrm{LI}=\mathrm{Lima}, \mathrm{LL}=$ La Libertad, $\mathrm{LO}=$ Loreto, $\mathrm{MD}=$ Madre de Dios, $\mathrm{MO}=$ Moquegua, $\mathrm{PA}=$ Pasco, $\mathrm{PI}=$ Piura, $\mathrm{PU}=$ Puno, $\mathrm{SM}=$ San Martín, $\mathrm{TA}=$ Tacna, $\mathrm{TU}=$ Tumbes, UC $=$ Ucayali.
Taxonomía

Ipomoea L., Sp. Pl. 1: 159 (1753)

Tipo: Ipomoea pes-tigridis L., lectotipo designado por Verdc., F1. Trop. Afr. Convolvulaceae: 108 (1963): BM

Etimología: Del griego ips, gen. ipós $(1 \psi)=$ gusano, larva, y omoios $($ o $\mu$ oro $\varsigma)=$ semejante.

Clave para las especies de Ipomoea del departamento de Lambayeque y zonas aledañas:

1. Ovario 3-locular. Estigmas trilobulados. Cápsula con 3-6 semillas . 2 Ovario 2 o 4-locular. Estigmas bilobulados. Cápsula con 4 semillas 4

2. Plantas perennes, robustas, con tallos estoloníferos, rastreros radicantes en los nudos. Sépalos exteriores de $23 \times 7 \mathrm{~mm}$, ovadolanceolados, largamente acuminados, cubiertos de pelos blancos sedosos. Sépalos interiores de 16x4 mm, largamente lanceolados, glabros. Pétalos de $60-85 \mathrm{~mm}$ de largo .......... I. indica

- Plantas anuales no estoloníferos. Sépalos con otras formas y medidas. Pétalos de 40-60 mm de largo 3

3. Sépalos iguales de $15-30 \mathrm{~mm}$, con largo acumen, lineal-lanceolados, zona proximal cubierta con abundantes pelos de base ensanchada. Pétalos de 40-55 mm de longitud. I. nil

- Sépalos desiguales; los exteriores de 8-13×2.5-4 $\mathrm{mm}$, sin acumen, lanceolados o elípticos, zona proximal cubierta con pelos cortos y pelos más largos de base ensanchada; los interiores de $12 \times 2.5 \mathrm{~mm}$, sutilmente lanceolados, glabros. Pétalos de hasta $60 \mathrm{~mm}$ de largo.. I. purpurea

4. Ovario 4-locular.

- Ovario bilocular ......................................

5. Corola tubular pero acampanada en el ápice, blanca, parte interna del tubo de color violeta. Pétalos de 20-28 mm de largo ..... I. piurensis

- Corola hipocrateriforme, rojo o anaranjado. Pétalos de 20-40 mm de largo 6

6. Láminas foliares profundamente pinnatisectas, ovadas o elípticas, base trunca. Corola rojo, de 20-25 mm de largo, el limbo con 5 lóbulos profundos, deltados, obtusos ...... I. quamoclit - Láminas foliares enteras o 3-5-lobadas pero no pinnatipartidas, ovada, base cordiforme o sagitada. Corola rojo intenso o rojo anaranjado de 25-40 $\mathrm{mm}$ de largo, el limbo con 5 lóbulos obtusos, pocos profundos

I. hederifolia 
7. Botones florales y corolas de pubescentes a tomentosas, por lo menos en la parte superior de las áreas mesopétalas

- Botones florales y corolas completamente glabros

8. Plantas arbustivas o subarbustivas, nunca volubles. Pétalos de 80-100 mm de largo ....... I. carnea

- Plantas volubles, rastreras o decumbentes. Pétalos de 30-40 mm de largo .... I. parasitica

9. Plantas decumbentes o rastreras, no volubles pero flexuosas en las ramas superiores....... 10

- Plantas volubles ........................................... 11

10. Láminas sub-reniformes, sub-truncadas o ligeramente cordadas en la base, de 30-90 $\mathrm{mm}$ de largo, más anchas que largas. Corola acampanada o infundibuliforme, blanca. Pétalos de hasta $50 \mathrm{~mm}$ de largo I. asarifolia

- Láminas profundamente cordadas en la base, con las aurículas divergentes agudas, de 125 $\mathrm{mm}$ de largo, más largas que anchas. Corola campanulada, rosada y con bandas más oscuras. Pétalos de hasta $70 \mathrm{~mm}$ de largo. I. incarnata

11. Pétalos de 8 a $10 \mathrm{~mm}$ de largo. Sépalos coriáceos o membranosos, raramente herbáceos, glabros 12

- Pétalos de 20a $120 \mathrm{~mm}$ de largo. Sépalos herbáceos, sub-escariosos, membranáceos o cartáceos, glabros o ligeramente puberulentos............ 14

12 Semillas pardo-ferrugíneas, pubescentes, cubierta de pelos largos, no opacas, ni brillantes, seríceas en los bordes. Tallos cilíndricos o angulosos, glabros, lisos o muricados I. amnicola

- Semillas marrón oscuras, glabras, opacas y brillantes. Tallos cilíndricos o angulosos, glabros a hirsutos, raramente puberulentos . .13

13. Sépalos exteriores elípticos a sub-lanceolados, con 1 o 3 nervaduras prominentes, glabros o pilosos. Sépalos interiores anchamente elípticos, glabros, obtusos I. batatas

- Sépalos exteriores ovado-lanceolados, sin nervaduras prominentes, glabros. Sépalos interiores ovados, glabros, agudos.. I. tiliacea

14. Corola hipocrateriforme de $70-120 \mathrm{~mm}$ de largo, blanco, el tubo verde claro en su interior, el limbo con 5 líneas verde claro formando una estrella. Estambres exertos I. alba

- Corola infundibuliforme a campanulada, de 10-70mm de largo, purpúrea, rojo-purpúrea, azul o blanca. Estambres insertos .. 15

15. Sépalos 3 exteriores y 2 interiores, con el dorso tuberculado en la vena media, mostrando una prominencia, de 2,5-4 $\mathrm{mm}$ de largo. Pétalos de $15-25 \mathrm{~mm}$ de largo I. aristolochiifolia
- Sépalos 2 exteriores y 3 interiores, dorso no tuberculado en la vena media, de $3.5-9 \mathrm{~mm}$ de largo. Pétalos de 16-70 mm de largo ........... 16

16. Láminas simples, enteras, a veces trilobadas, acuminadas, base profundamente cordadas, aurículas redondeadas, glabras en el haz y el envés, de 20-100x20-90 mm. Pétalos de 16-30 $\mathrm{mm}$ de largo....

I. dumetorum

- Láminas palmaticompuestas, agudas, mucronuladas, no presentan base profundamente cordadas, ni aurículas, glabras sólo en el envés, de 20-100 x 20-120 mm. Pétalos de 17-70 mm de largo ......

17. Láminas 5-palmatisectas, foliolos ovados o suborbiculares, a veces agudos, mucronulados, glabros en el envés, en el haz con pelos cortos en los márgenes y vena media, de 2-100 x 20-90 $\mathrm{mm}$. Pétalos de 35-70 $\mathrm{mm}$ de largo. I. cairica Láminas 5-palmatipartidas, con algunos foliolos subdivididos, foliolos linear-lanceolados, agudos, mucronulados, glabros, de 50x120 mm. Pétalos de 17-25 mm de largo ........ I. wrightii

\section{Subgénero Ipomoea}

Serie Pharbitis (Choisy) D.F.Austin, Taxon 29(4): 501 (1980)

$\equiv$ Pharbitis Choisy, Mém. Soc. Phys. Genève 6(2): 438 (1834) [basión.]

Tipo: Pharbitis hispida (Zuccagni) Choisy, Convolv. Orient. 56 (1833)

1. Ipomoea purpurea (L.) Roth, Bot. Abh. Beobacht.: 27 (1787)

三Convolvulus purpureus L. Sp. Pl. (ed. 2)

1: 219-220 (1762) [basión.]

= Ipomoea glandulifera Ruiz \& Pav., Fl.

Peruv. 2: 12, t. 121 (1799)

= Ipomoea hirsutula J. Jacq., Ecl. Pl. Rar. 1(5-6): 65, pl. 44 (1811)

= Ipomoea diversifolia Lindl., Edwards's

Bot. Reg. 23: pl. 1988 (1837)

Ind. loc.: no especifica

Lectótipo designado por Austin in Ann. Missouri Bot. Gard. 62: 193 (1975): G

Etimología: Del latín "purpureus": refiriéndose al color púrpura de las flores.

Nombre vernáculo local: "don diego de día", "auroras", "campanilla morada", "campanillas" y "yedra morada". 


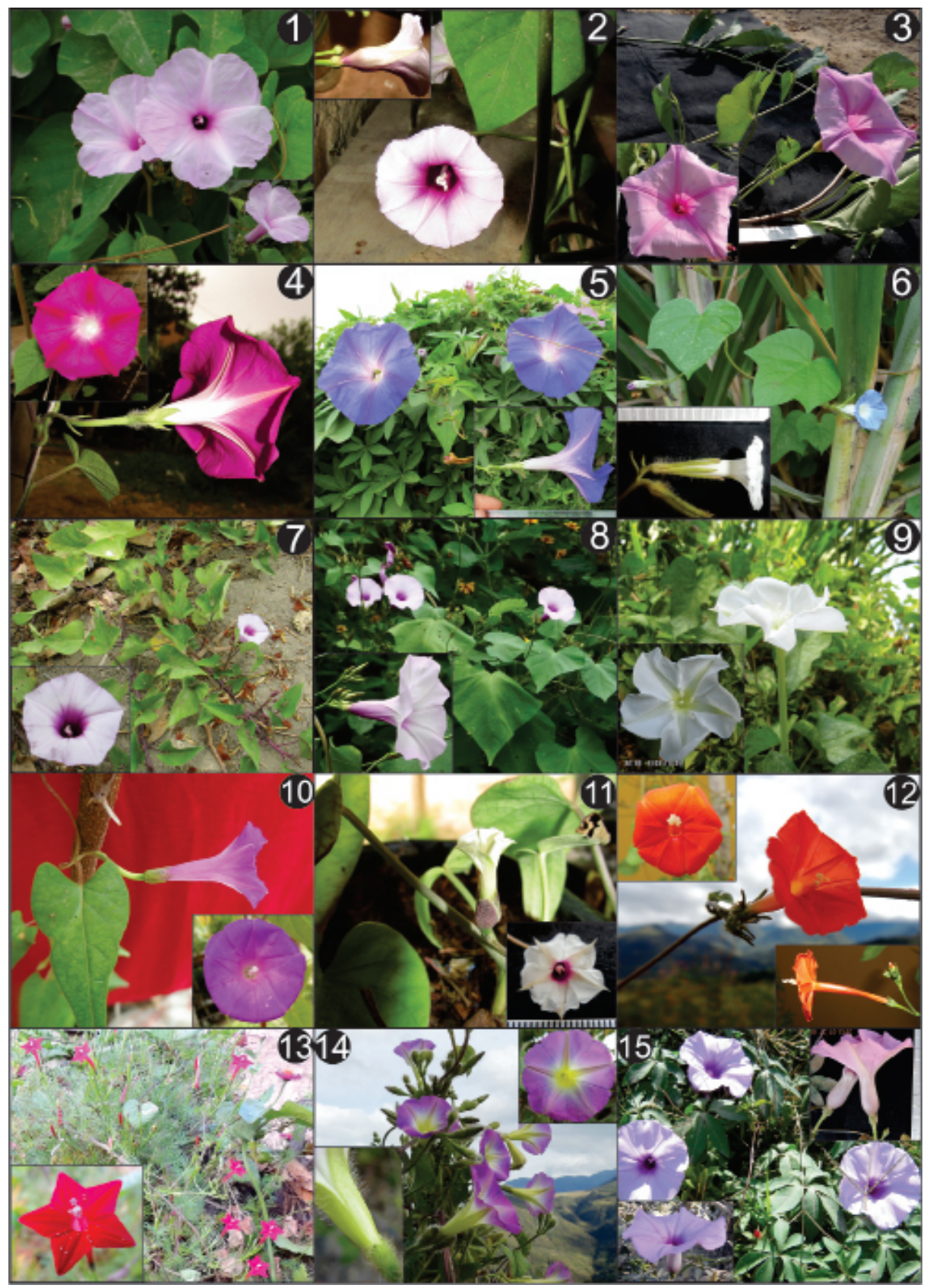

Figura 2a. Taxones reportados para Ipomoea colectados en el departamento de Lambayeque y zonas aledañas (Perú). 1. Ipomoea carnea, 2. I. amnicola, 3. I. incarnata, 4. I. purpurea, 5. I. indica, 6. I. nil, 7. I. batatas, 8. I. tiliacea, 9. I. alba, 10. I. dumetorum, 11. I. piurensis, 12. I. hederifolia, 13. I. quamoclit, 14. I parasitica, 15. I. cairica. Flowers of Ipomoea in Lambayeque department and surrounding areas (Peru). 


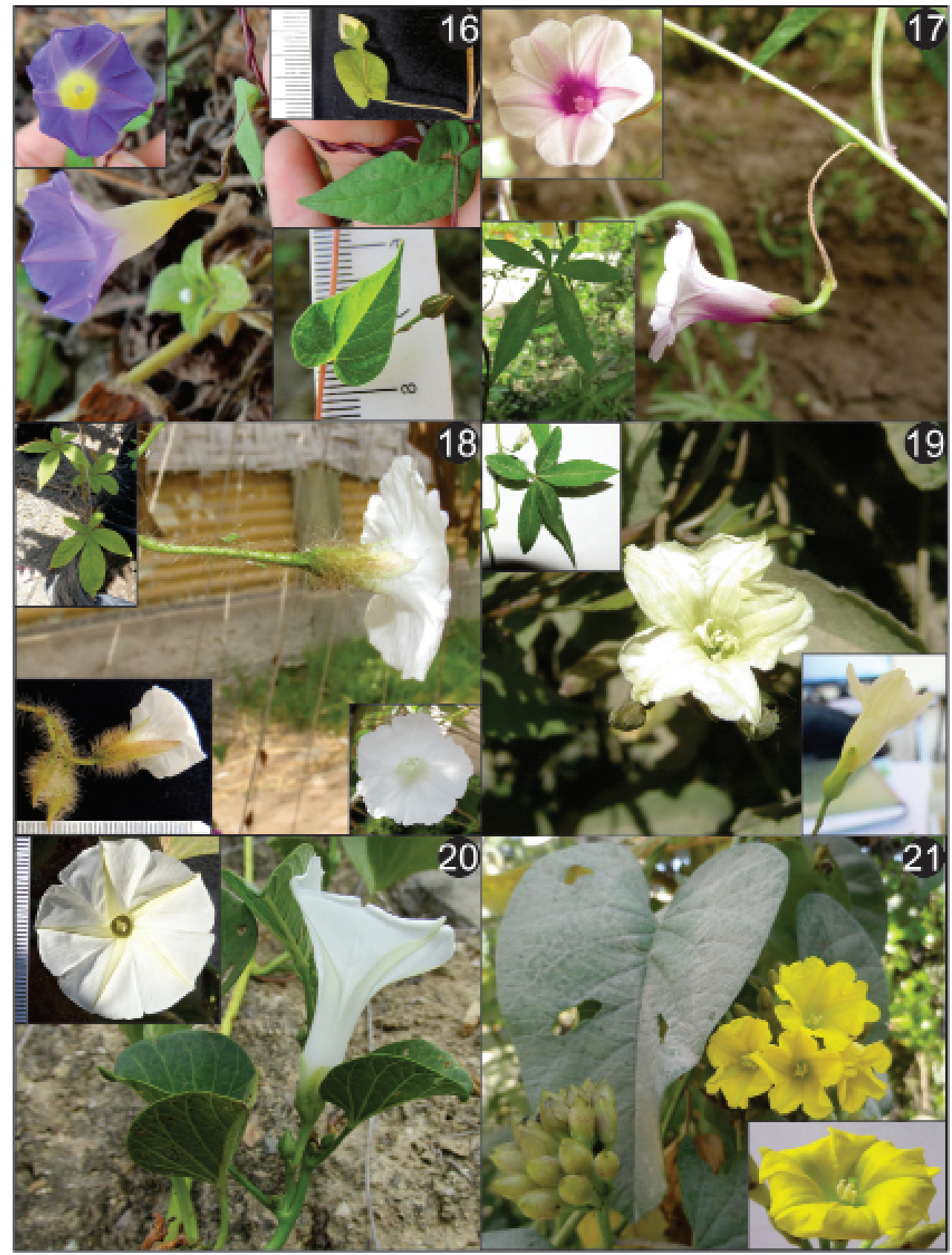

Figura2b. Taxones reportados para Ipomoea y Merremia colectados en el departamento de Lambayeque y zonas aledañas (Perú). 16. I. aristolochiifolia, 17. I. wrightii, 18. Merremia aegyptia, 19. M. quinquefolia, 20. I. asarifolia y 21. M. umbellata. Flowers of Ipomoea and Merremia in Lambayeque department and surrounding areas (Peru). 
Distribución: De origen americano. EE.UU., ME, NI, DO, HA, PN, CO, VE, EC, BR, BO, AR, PAR y PE (AP, AY, CU, HU, JU, LI, LL, PA, SM, TA, TU, CA, LA).

Rango altitudinal: desde los 1000 a 2500 msnm (Brako \& Zarucchi, 1993), donde se encontró desde los 18 a 1554 msnm.

Hábitat: Bosques secos y comunidades de Cactáceas (Galán de Mera et al., 2015)

Usos: Ornamental y densas matas de cercos

Propagación: Semillas y vegetativa mediante esquejes

Conservación: Cosmopolita. Se encuentra en el Área de Conservación Regional Comunal Bosque Moyán-Palacios, Área de Conservación Privada Chaparrí, Santuario Histórico Bosque de Pomac y Refugio de Vida Silvestre Bosques Nublados de Udima.

Fenología: Agosto a abril.

Material estudiado Nuevo reporte para LA y CA

Lambayeque: Lambayeque. Jardines de Lambayeque, aprox. 6²'23.88'S 7954'13.77'O, 18 m.s.n.m., 10-IV-2013, L. Alarcón \& G. Torres, PRG 14519. Motupe, Huerto Frutícola, A. Díaz, PRG 4062. Mochumí, A. Díaz \& L. Cerna, PRG 4478. Chiclayo. Oyotún, J. Laos, PRG 6737. Reque, S. Llatas, PRG 6073; Luya, A. Díaz \& L. Cerna, PRG 417. Zaña, C. Abad, J. Escurra, E. Benavides \& S. Llatas, PRG 6770. Chongoyape, C. Abad, PRG 6745. Chongoyape, C. Abad, J. Laos \& S. Llatas, PRG 6754. Cajamarca: Santa Cruz. Borde de camino saliendo de Udima y entrando al Caserío Maraypampa, aprox. 6 50'58.37'S 7905'38.45"O, 1554 m.s.n.m., 24-VIII-2013, L. Alarcón, G. Torres \& B. Esquerre, PRG 14529. Borde de caminos en Caserío Udima, 1590 m.s.n.m., 24-VIII-2013, L. Alarcón, G. Torres \& B. Esquerre, PRG 14738.

Serie Heterophyllae (House) Austin, Taxon 29(4): 501 (1980).

三 Subsección Heterophyllae House, Ann. New York Acad. Sci. 18: 194 (1908)

Tipo: Ipomoea heterophylla Ortega, Nov. Pl. Descr. Dec. 1:9 (1797).
2. Ipomoea indica (Burm.) Merr., Interpr. Herb. Amboin. 445 (1917)

= Convolvulus indicus Burm., Herb. Amboin. Auctuar. 7: [6] (1755)[basión.]

= Ipomoea acuminata (Vahl) Roem \& Schult., Syst. Veg. 4: 228 (1819), comb. inval., non Ruiz \& Pav., Fl. Peruv. 2, tab. 120fig. b (1799)

= Ipomoea congesta R. Br., Prodr. 485 (1810)

Ind. loc.: "Habitat in America"

Lectótipo designado por Fosberg in Bot. Not. 129: 35-38 (1976): Besler, Hort. Eystett. Ordo n. ${ }^{\circ} 13$, fol. 8 fig. 2 (1613)

Etimología: Del latín “indicus": refiriéndose a la India o del Este de las Indias.

Nombre vernáculo local: "campanilla", "suspiros", "batatilla lila".

Distribución: Pantropical, EE.UU., BH, CU, JA, DO, HA, ME, Las Antillas, BE, GU, NI, CR, PN, CO, VE, GU, SUR, GUF, BR, BO, UR, AR, CH y PE (AM, CA, LA, LI, PA).

Rango altitudinal: 0 a $2000 \mathrm{msnm}$ (Brako \& Zarucchi, 1993), donde se pudo encontrar entre los 18-20 msnm.

Hábitat: Bosques secos y comunidades de Cactáceas

Usos: Ornamental

Propagación: Semillas y vegetativamente mediante esquejes

Conservación: Cosmopolita. No se encuentra en ninguna área protegida

Fenología: Florece durante todo el año.

Observaciones: McDonald (1994) menciona que Ipomoea indica es una de las especies más variable del género. A altitudes mayores se caracteriza por tener inflorescencias aglomeradas, hojas canescentes en el envés y sépalos lanceolados con pelos adpresos, pero a altitudes más bajas tiene inflorescencias más abiertas, hojas glabras y los sépalos más anchos y glabros. Existe variación clinal de la especie en todos los territorios que tienen gradientes altitudinales, por lo tanto se utiliza un concepto politípico. Carranza (2007) también comenta 
que la especie tiene cierta variabilidad sobre todo en la pubescencia de los sépalos y en las hojas; las hojas de los tallos jóvenes generalmente son de enteras a lobuladas, y las de los tallos más viejos profundamente trilobadas.

Material estudiado Reportada para LA y CA (Brako \& Zarucchi, 1993)

Lambayeque: Chiclayo. Fundo la Joyita, Pimentel, aprox. 647'53.05'S 7953'05.29”O, 20 m.s.n.m., 03-IV-2013, L. Alarcón \& G. Torres, PRG 14512. Reque, J. Laos \& S. Llatas, PRG 3449.

3. Ipomoea nil (L.) Roth, Catal. Bot. 1: 36 (1797)

=Convolvulus nil L., Sp. P1. 1. 219 (1762)

= Batatas setosa (Ker Gawl.) Lindl., Sketch

Veg. Swan R.: 15 (1839)

= Calonyction campanulatum Hallier f., Bull. Herb. Boissier 5(12): 1050, pl. 18, f. 2 (1897)

= Calonyction pavonii (Choisy) Hallier f., Bull. Herb. Boissier 5(12): 1048, pl. 17, f. 4-5; pl. 18, f.1. (1897).

Ind. loc.: "Habitat in America"

Lectótipo designado por Verdc. in Taxon 6: 232-233 (1957)

Etimología: Del árabe "nil": nombre adoptado por los primeros europeos que cultivaron esta especie (Shinners, 1965)

Nombre vernáculo local: "campanita", "campanilla".

Distribución: Pantropical. EE.UU., BH, CU, DO, HA, ME, BE, GU, NI, CR, PN, CO, VE, GU, SUR, BR, BO, AR y PE (AY, CA, CU, HU, JU, LA, LI, LO, PI, SM, TU, LL).

Rango altitudinal: desde los 0 a $1500 \mathrm{msnm}$ (Brako \& Zarucchi, 1993).

Hábitat: Bosques secos y comunidades de Cactáceas

Usos: Ornamental

Propagación: Semillas

Conservación: Ampliamente distribuida en poblaciones estables. Se encuentra en el Área de Conservación Regional Comunal Bosque Moyán-Palacios y Área de Conservación
Privada Chaparrí.

Fenología: Florece durante todo el año

Material estudiado Reportada para LA y CA

(Brako \& Zarucchi, 1993)

Lambayeque: Lambayeque. Olmos, La Beatita, J. Laos, L. Vásquez \& G. Delgado, PRG 4924. Olmos, La Beatita, C. Abad, J. Laos \& S. Zelada, PRG 6776. Portachuelo, Olmos, A. Díaz \& L. Vásquez, PRG 294. Portachuelo, Olmos, L. Cerna \& C. Stein, PRG 1125. Olmos, La Granja-Km 89, A. Díaz, PRG 1920. Olmos, R. Ferreyra, USM 9085. Motupe, Sapotal, J. Laos \& S. Llatas, PRG 5012. Motupe, Huerto frutícola, A. Díaz, PRG 4063. Motupe, Quebrada del Tabacal, C. Abad \& S. Llatas, PRG 6758. Chiclayo. Espinal, A. Díaz, PRG 3111. Bordes de cultivos de caña de azúcar en Pucalá. aprox. 646’31.18'S 79³6’35.72”O, 84 m.s.n.m., 07-IV-2013, L. Alarcón, G. Torres, C. Rojas \& B. Esquerre, PRG 14515. Oyotún, J. Laos, PRG 6736. Chongoyape, L. Vásquez, PRG 2174. Chongoyape, La Puntilla, C. Abad \& J. Laos, PRG 6779. Cajamarca: Chota. Pasando Potrerillo, aprox. 6'29'22.50"'S 7908'07.68'O, 1438 m.s.n.m., 23-VI-2013, C. Rojas, B. Esquerre \& R. Cavero, PRG 14522. Macuaco, C. Abad \& L. Vásquez, PRG 6749. Bordes de carretera, pasando Potrerillo, 1330 m.s.n.m. 23-VI-2013, C. Rojas, B. Esquerre \& R. Cavero, PRG 14739.

Subgénero Eriospermum (Hallier f.) Verdc., Taxon 6: 152 (1957)

= Sección Eriospermum Hallier f., Bot. Jahrb. Syst. 18: 149 (1893)

Tipo: Ipomoea digitata L., Syst. Nat. (ed. 10) 2: 924 (1759)

Sección Eriospermum Hallier f., Bot. Jahrb. Syst. 18: 149 (1893)

Serie Jalapae (House) D.F.Austin, Taxon 29(4): 502 (1980)

三 Subsección Jalapae House, Ann. New York Acad. Sci. 18(6): 224 (1908) [basión.] Tipo: Ipomoea jalapa (L.) Pursh., Fl. Amer. Sept. 146-147 (1813-1814)

4. Ipomoea amnicola Morong, Ann. New York Acad. Sci. 7: 170 (1892) 
= Ipomoea nuda N.E. Br., Trans. Bot. Soc.

Edinburgh 20: 63 (1894)

Holótipo: NY

Etimología: Deriva del latín "amnis" (río) + "colo" (cultivar): refiriéndose al hábitat de la especie el borde de los ríos.

Nombre vernáculo local: No conocido

Distribución: EE.UU, ME, CO, BR, BO, PAR, AR y PE (CA)

Rango altitudinal: $970 \mathrm{msnm}$.

Hábitat: Bosques secos y comunidades de Cactáceas

Usos: Eventualmente como ornamental

Propagación: Semillas

Conservación: Se encuentra en el Refugio de Vida Silvestre Bosques Nublados de Udima.

Fenología: Marzo a mayo.

Material estudiado Reportada para CA (Brako \& Zarucchi, 1993)

Cajamarca: Santa Cruz. Carretera a Catache, saliendo de Chongoyape, aprox. 6 39'18.58'S 7904'29.33”O, 970 m.s.n.m., 24-VIII-2013, L. Alarcón, G. Torres \& B. Esquerre, PRG 14727.

5. Ipomoea carnea Jacq., Enum. Syst. Pl. 13 (1760)

= Ipomoea carnea f. albiflora Moldenke, Phytologia 2: 224 (1947)

= Ipomoea crassicaulis (Benth.) B.L. Rob., Proc. Amer. Acad. Arts. 51)10): 530 (1916) $\equiv$ Batatas crassicaulis Benth., Bot. Voy. Sulphur 134 (1845) [basión.]

= Ipomoea fistulosa Mart ex Choisy, Prodr. 9: 349 (1845)

Ind. loc.: no especifica

Lectótipo designado por Austin in Taxon 26(2-3): 237 (1977): Taxon 26(2-3): 236, Fig. 1

Etimología: Del latín "carneus": refiriéndose al color carne de la flor.

Nombre vernáculo local: "borrachera", "algodón bravo", "campanilla morada", "camote caspi", "campanola", "mata caballo".

Distribución: Sur de EE.UU., DO, HA, JA, ME, GU, NI, CR, PN, CO, VE, GU, BR, BO, PAR y PE (AM, AP, CA, CU, LI, LO, PI,
TU, LA).

Rango altitudinal: 0 a 2000 msnm (Brako \& Zarucchi, 1993), pero se encontró entre 23 a $670 \mathrm{~m}$.

Hábitat: Bosques secos y comunidades de Cactáceas

Usos: Ornamental, cubriendo los campos de color rosa durante la floración; es peligrosa para el ganado por su alta toxicidad provocando la llamada 'borrachera' (embriaguez).

Propagación: Semillas y esquejes

Conservación: Ampliamente distribuida y con poblaciones estables. Se encuentra en el Área de Conservación Regional Comunal Bosque Moyán-Palacios, Santuario Histórico Bosque de Pómac y Área de Conservación Privada Chaparrí.

Observaciones: La especie relacionada I. carnea subsp. carnea, es una liana con poblaciones en América del Sur.

Fenología: Agosto a mayo.

Material estudiado Nuevo reporte para LA, reportada para CA (Brako \& Zarucchi, 1993)

Lambayeque: Lambayeque. Las Dunas, Carretera Chiclayo-Lambayeque, aprox. 6 42 ' 49.57'S 7954'01.76"O, 23 m.s.n.m., 10-IV-2013, L. Alarcón, G. Torres \& B. Esquerre, PRG 14518. Lambayeque, G. Alfaro \& L. Vásquez, PRG 1928. Yéncala, R. Ferreyra, USM 7617. Chiclayo. Reque, N. Angulo \& A. López. HUT 422. Reque, C. Abad \& S. Llatas, PRG 6768. Cajamarca: Santa Cruz. Carretera Cumbil-Catache, aprox. 6036'46.47'S 79¹3'35.40"O, 410 m.s.n.m, 24-VIII-2013, L. Alarcón, G. Torres \& B. Esquerre. PRG 14737.

6. Ipomoea incarnata (Vahl) Choisy, Prodr. 9: 360 (1845)

$\equiv$ Convolvulus incarnatus Vahl, Eclog. Amer. 2: 12 (1798) [basión]

= Ipomoea monosperma Sprengel ex Choisy, Prodr. 9: 360 (1845)

= Ipomoea kinbergii Andersson, Kongl. Svenska Vetensk. Acad. Handl., n.s: 212 (1853)

Ind. loc.: "In Curacao" 
Sintipo: C

Etimologia: Del latín "incarnatus": refiriéndose al color encarnado de la flor.

Nombre vernáculo local: "bejuco"

Distribución: Norte y Noroeste de Sudamérica, Islas Galápagos, BR, CO, VE, EC y PE (LI, PI, TU, LA).

Rango altitudinal: 0 a 2000 msnm (Brako \& Zarucchi, 1993). Se encontró a $245 \mathrm{~m}$

Hábitat: Bosques secos y comunidades de Cactáceas, vegetación de riberas

Usos: Eventualmente como ornamental

Propagación: Semillas

Conservación: Se encuentra en el Área de Conservación Regional Comunal Bosque Moyán-Palacios y Área de Conservación Privada Chaparrí.

Fenología: Julio a agosto.

Material estudiado Nuevo reporte para LA Lambayeque: Lambayeque. Olmos, A. Díaz \& L. Vásquez, PRG 309. Olmos-Portachuelo, L. Cerna \& O. Stein, PRG 1121. Chiclayo. Espinal, L. Vásquez, PRG 3072.

Las Delicias - Puente del río Zaña, saliendo de Oyotún y llegando a Pan de azúcar, aprox. 6047'37.77' S 79¹7'36.87" O, 245 m.s.n.m., 27-VII-2013, L. Alarcón, G. Torres \& B. Esquerre. PRG 14528. Chongoyape, Tinajones, A. Díaz \& L. Cerna, PRG 1279. Chongoyape, Tinajones, L. Vásquez \& H. Ocampo, PRG 1845. Chongoyape, Tinajones, A. Díaz \& L. Cerna, PRG 424. Chongoyape, Tinajones, C. Abad, J. Laos \& S. Llatas, PRG 6756. Chongoyape, Tinajones, S. Llatas, HUT 16543.

Serie Batatas (Choisy) D.F. Austin, Taxon 45(1): 13 (1996)

$\equiv$ Batatas Choisy, Mém. Soc. Phys. Genève 6(2): 434-435 (1834)

Tipo: Batatas edulis (Thunb.) Choisy, lectótipo designado por Roberty in Boissiera 10. 147 (1964).

7. Ipomoea batatas var. apiculata (M. Martens \& Galeotti) J.A. McDonald \& D.F. Austin in Brittonia 42(2): 118 (1990).

Ipomoea apiculata $\mathrm{M}$. Martens \& Galeotti in Bull. Acad. Roy. Sci. Bruxelles 12(2): 262
(1845)

= Ipomoea apiculata M. Martens \& Galeotti in Bull. Acad. Roy. Sci. Bruxelles 12(2): 262 (1845) [basión.]

Ind. loc.: "Mexico: dans les dunes de Ver-Cruz"

Holótipo: BR; isótipo: $\mathrm{P}$

Etimología: Del latín "apiculatus", refiriéndose a que se encuentra apiculado, cubierto con un punto.

Nombre vernáculo local: "camote"

Distribución: Pantropical de origen americano. EE.UU, ME, AN, CO, PAR, CU, AR, BO y PE (AM, CA, IC, JU, LI, LO, SM, $\mathrm{HU})$.

Rango altitudinal: Desde los 0 a 2500 msnm (Brako \& Zarucchi, 1993).

Hábitat: Bosques secos y comunidades de Cactáceas

Usos: Las raíces tuberosas en la alimentación humana y animal y los tallos y hojas como forraje de animales domésticos.

Propagación: Esquejes

Conservación: Planta cultivada con amplia variación genética que se conserva en el Banco de Germoplasma del Centro Internacional de la Papa (CIP, Perú).

Fenología: Julio a abril.

Material estudiado Nuevo reporte para LA, reportada para CA (Brako \& Zarucchi, 1993)

Lambayeque: Lambayeque. Bordes de cultivos de loche en Mórrope, aprox. 6 ${ }^{\circ} 32$ '52.23's 8000'59.93”O, 20 m.s.n.m., 22-XI-2013, PRG 14726.

8. Ipomoea tiliacea (Willd.) Choisy, Prodr. 9: 375 (1845)

三Convolvulus tiliaceus Willd., Enum. Pl.

1: 203-204 (1809) [basión.]

三Convolvulus fastigatus Roxb., Hort.

Bengal.: 13 (1824)

= Ipomoea fastigiata (Roxb.) Sweet, Hort.

Brit. 1: 288 (1826) nom. nud.

Ind. loc.: "In Brasilia"

Holótipo: B-W-3691 
Etimología: Del latín “tiliaceus”, derivado del género Tilia, género tipo de la familia Tiliaceae, conocido como el árbol de tilo.

Nombre vernáculo local: "batatilla", "bejuco blanco", "bejuco de puerco", "quiebraplatos", "campanilla", "batata".

Distribución: América tropical; BH, DO, HA, JA, AN, ME, GU, NI, CR, PN, PR, CO, VE, GUF, SUR, GU, BR y PE (JU, LO, PI, LL, LA, CA).

Rango altitudinal: 0 a $500 \mathrm{msnm}$

Hábitat: Bosques secos y comunidades de Cactáceas

Usos: Por formar raíces fibrosas engrosadas está siendo utilizada en programas de mejoramiento genético del camote.

Propagación: Semillas y vegetativa

Conservación: Se encuentra en el Área de Conservación Regional Comunal Bosque Moyán-Palacios, Área de Conservación Privada Chaparrí y Refugio de Vida Silvestre Bosques Nublados de Udima.

Fenología: Septiembre a julio.

Material estudiado Nuevo reporte para LA y CA

Lambayeque. Lambayeque, Olmos-La Beatita, L. Vásquez, J. Laos, G. Delgado \& S. Llatas, PRG 4037. Bordes de carretera Chiclayo-Olmos, antes del puente El Silencio - Km 17, aprox. 5056'06.15"S 79³5'32.58”O, 536 m.s.n.m., 13-IV-2013, L. Alarcón, G. Torres \& B. Esquerre, PRG 14729. Chiclayo. Espinal, C. Abad \& S. Llatas, 6763 PRG. Oyotún, J. Laos, PRG 6678. Río Zaña, N. Angulo, HUT 1087. Cajamarca. Santa Cruz. El Huaro, saliendo de Florida, aprox. 6051'57.84'S 7907'07.47'O, 927 m.s.n.m., 27-VII-2013, L. Alarcón, G. Torres, B. Esquerre \& R. Cavero, PRG 14730.

Sección Erpipomoeae Choisy, Mém. Soc. Phys. Genève 6: 444 (1833)

Tipo: Ipomoea pes-caprae (L.) R.Br., Narr. Exped. Zaire 477 (1818)

9. Ipomoea asarifolia (Desr.) Roem. \& Schult., Syst. Veg. 4: 251 (1819) 三Convolvulus asarifolius Desr., Encycl. 3:
562 (1789)[basión.]

= Ipomoea crassifolia Cav., Descr. P1. 100 (1802)

= Ipomoea urbica Choisy, Prodr. 9: 349(1845)

Ind. loc.: "Ad Senegal, Roassillon"

Holótipo: P-JUSS.

Etimologia: De las palabras en latín "Asarum" (Género de la familia Aristolochiaceae) + "folium" (hojas): refiriéndose al parecido de la forma de las hojas de Asarum, especie conocida como el "jengibre salvaje".

Nombre vernáculo local: "galán de día", "bejuco"

Distribución: BR, JA, PN, CO, VE, EC, BO, PAR y PE (LA, PI, TU, LL).

Rango altitudinal: 84 a $266 \mathrm{~m} .0$ a 500 msnm (Brako \& Zarucchi, 1993)

Hábitat: Bosques secos y comunidades de Cactáceas

Usos: Sus tallos largos para atar maderos en construcciones ligeras. Por su crecimiento acelerado en suelos degradados es posible su utilización en la recuperación de suelos.

Propagación: Semillas y ocasionalmente mediante tallos radicantes.

Conservación: Se encuentra en el Área de Conservación Regional Comunal Bosque Moyán-Palacios, Área de Conservación Privada Chaparrí y Santuario Histórico Bosque de Pomac.

Fenología: Febrero a agosto.

Material estudiado Reportada para LA (Brako \& Zarucchi, 1993)

Lambayeque: Lambayeque. Bordes de caminos en Caserío Zapote, aprox. 607' 52.09"'S 7943'24.51" O, 136 m.s.n.m, 06-IV-2013, L. Alarcón \& G. Torres. PRG 14514. Lambayeque, L. Vásquez \& H. Ocampo, PRG 1443. Lambayeque, El Ciénago, L, Vásquez, L. Cerna \& A. Pisfil, PRG 068. Mochumí, Vivero Frutícola, C. Abad, PRG 6759. Olmos, N. Angulo, HUT 2089. Jayanca, R. Ferreyra, USM 9078. Ferreñafe. Pítipo, C. Abad, PRG 6772. Chiclayo. Chongoyape, Tinajones, C. Abad, J. Laos \& S. Llatas, PRG 6753. Pátapo, Tulipe, R. Ferreyra, USM 7609. 
Subgénero Quamoclit (Moench) Clark, Fl. Brit. India 4: 198 (1883)

=Quamoclit Mill., Gard. Dict. Abr. 4 (1754)

Tipo: Ipomoea quamoclit L., Sp. Pl. 1: 159-160 (1753): BM

Sección Calonyction (Choisy) Griseb, Fl. Brit.

W. I. 466 (1864[1862])

E Calonyction Choisy, Mém. Soc. Phys. Genève 6(2): 441 (1834)

Tipo: Calonyction speciosum Choisy, Mém. Soc. Phys. Genève 6(2): 441 (1834)

10. Ipomoea alba L. Sp. P1. 1: 161 (1753)

= Convolvulus aculeatus L., Sp. Pl. 1: 155 (1753)

= Convolvulus bona-nox (L.) Spreng., Syst.

Veg. 1: 600 (1825)

=Ipomoea bona-nox (L.) Spreng., Syst. Veg. 1: 600 (1825) [basión.]

Ind. loc.: "Habitat in Malabariae arenofis" Lectótipo designado por Verdc. in Fl. Trop. E. Africa, Convolvulaceae: 130 (1963)

Etimología: Del latín “albus”, refiriéndose al color blanco de sus flores.

Nombre vernáculo local: "galán de noche", "bejuco", "buenas noches", "trompeta", "dama de noche".

Distribución: América tropical y sub tropical. EE.UU., BH, CU, DO, HA, ME, BE, GU, HO, NI, CR, PN, CO, VE, SUR, GUF, BR, BO, AR y PE (AM, AY, CA, CU, HU, JU, LA, LI, LL, LO, MD, SM, UC).

Rango altitudinal: 0 a $1500 \mathrm{msnm}$

Hábitat: Bosques secos y comunidades de Cactáceas

Usos: Ornamental y reforzamiento de cercos en terrenos de cultivo

Propagación: Semillas y vegetativa mediante esquejes

Conservación: Se encuentra en el Área de Conservación Regional Comunal Bosque Moyán-Palacios, Santuario Histórico Bosque de Pomac y Área de Conservación Privada Chaparrí.
Fenología: Setiembre a julio.

Observaciones: A diferencias de otras especies sus flores se abren durante la noche y se cierran con los primeros rayos de sol.

Material estudiado Reportada para LA y CA (Brako \& Zarucchi, 1993)

Lambayeque: Ferreñafe. Orillas del Río La Leche, Puente Colgante Pítipo cerca de Santa Clara, aprox. 6²6.841'S 7944.001'O, 84 m.s.n.m., 13-VII-2013, L. Alarcón, G. Torres \& B. Esquerre, PRG 14527. Chiclayo. Espinal, C. Abad, PRG 6765. Zaña, L. Vásquez, H. Ocampo \& R. Tapia, PRG 1449. Reque, S. Llatas, PRG 6064. Reque, J. Laos, S. Llatas \& H. Aurazo, PRG 2825.

Sección Exogonium (Choisy) Griseb., Fl. Brit. W. I. 472 (1864[1862])

EExogonium Choisy, Mém. Soc. Phys. Genève 6(2): 443 (1834)

Tipo: Exogonium bracteatum (Cav.) Choisy, Gen. Hist. 4:264 (1838) nom. illeg. hom.

11. Ipomoea dumetorum Willd. ex Roem. \& Schult., Syst. Veg. 4: 789 (1819)

= Convolvulus dumetorum Kunth, Nov. Gen. Sp. (quarto ed.) 3: 101 (1818)

= Convolvulus pulchellus Kunth, Nov. Gen. Sp. (quarto ed.) 3: 101 (1818)

= Convolvulus glaucescens Kunth, Nov. Gen. Sp. (quarto ed.) 3: 80 (1819)

= Convolvulus pauciflorus Willd. Ex Roem.

\& Schult., Syst. Veg. 4: 302 (1819)

Ind. loc.: "In America merid."

Holótipo: B; isótipo: P.

Etimología: Del latín “dumetum”, que significa "matorral", "chaparral", "maleza", "rastrojo", "boscaje"; refiriéndose al hábitat de esta especie. En ocasiones el epíteto específico es erróneamente mencionado como "dumetora".

Nombre vernáculo local: No conocido

Distribución: EE.UU., ME, CO, EC, AR, BO y PE (AM, AY, CU, JU, LI, LO, PA, TA, AP, AR, CA). 
Rango altitudinal: 0 a $3500 \mathrm{msnm}$.

Hábitat: Bosques secos y comunidades de Cactáceas

Usos: Eventualmente ornamental. Es considerada maleza de cultivos

Propagación: Semillas

Conservación: No se encontró en áreas naturales protegidas

Fenología: Setiembre a julio.

Material estudiado Nuevo reporte para CA Cajamarca: Chota: Borde de carretera pasando Maichil e Izco, aprox. 6029'38.51' 'S 7908'29.07'O, 1363 m.s.n.m., 23-VI-2013, C. Rojas, B. Esquerre \& R. Cavero, PRG 14525.

12. Ipomoea piurensis O'Donell, in Lilloa 26: 382, t. 13: 382 (1953)

= Ipomoea piurensis f. rosea O'Donell in Lilloa 26: 383 (1953)

Ind. loc.: "Perú: Piura, Pariñas Valley" Holótipo: US

Etimología: el nombre "piurensis" deriva del departamento de Piura en Perú.

Nombre vernáculo local: No conocido

Distribución: Norte de América del Sur. CO, VE, GU, EC, BR y PE (AM, CA, PI, LA). Rango altitudinal: 96 a $600 \mathrm{msnm}$.

Hábitat: Bosques secos y comunidades de Cactáceas

Usos: Cercos en terrenos de cultivo. Es también considerada maleza

Propagación: Semillas

Conservación: Se encuentra en el Área de Conservación Regional Comunal Bosque Moyán-Palacios, Santuario Histórico Bosque de Pomac y Área de Conservación Privada Chaparrí.

Fenología: Mayo a noviembre.

Material estudiado Nuevo reporte para LA, reportada para CA (Brako \& Zarucchi, 1993)

Lambayeque: Lambayeque. Olmos, A. Díaz \& L. Vásquez, PRG 295. Jayanca, N. Angulo, HUT 2453. Ferreñafe. Batangrande, A. Díaz, PRG 3711.
Pítipo, S. Llatas \& J. Laos, PRG 5465. Borde de camino a Santa Clara, antes del Puente Colgante Pítipo, aprox. 6²8'21.07's 7942'41.11'O, 96 m.s.n.m., 13-VII-2013, L. Alarcón, G. Torres \& B. Esquerre, PRG 14728. Chiclayo. Chongoyape, C. Abad, J. Laos \& S. Llatas, PRG 6757. Garraspiña Chongoyape, C. Abad \& J. Laos, PRG 6780.

Sección Mina (Cerv.) Griseb., Fl. Brit. W. I. $472(1864[1862])$

= Mina Cerv., Nov. Veg. Descr. 1: 3 (1824)

Tipo: Mina lobata Cerv., Nov. Veg. Descr. 1: 3-4 (1824)

13. Ipomoea hederifolia L., Syst. Nat. (ed. 10) 925 (1759)

= Ipomoea coccinea Sessé \& Moc., Sp. Pl. (ed. 2) 228 (1762)

= Ipomoea luteola Jacq., Collectanea 2: 266 (1788)

Ind. loc.: no especifica

Lectótipo designado por O'Donell in Lilloa 29: 48 (1959): LINN

Etimología: Del latín "hederacea" epíteto que significa "que se parece a Hedera", y "folium" referido a la forma de la hoja.

Nombre vérnaculo local: "yedra colorada" Distribución: Pantropical, EE.UU., DO, HA, JA, ME, GT, NI, CR, PN, CO, VE, GU, EC, BO, BR, PAR y PE (AY, CA, CU, JU, LA, LI, SM).

Rango altitudinal: 0 a 1335 msnm.

Hábitat: Bosques secos y comunidades de Cactáceas

Usos: Ornamental. Planta trepadora utilizada para cubrir muros, bases de columnas, pérgolas, entre otros.

Propagación: Semillas

Conservación: Se encuentra en el Área de Conservación Regional Comunal Bosque Moyán-Palacios y Área de Conservación Privada Chaparrí.

Fenología: Agosto a junio.

Material estudiado Reportada para LA y CA (Brako \& Zarucchi, 1993) 
Lambayeque: Lambayeque. Sapotal, Motupe, S. Llatas, PRG 5002. Motupe - quebrada del tabacal, C. Abad \& S. Llatas, PRG 6751. Chiclayo: Espinal, L. Vásquez, J. Laos \& G. Delgado, PRG 4546. La Jara - Oyotún, J. Laos \& H. Aurazo, PRG 5636. Oyotún, Laos S., PRG 6732. Bordes de cultivos de caña de azúcar en Pucalá, aprox. 6046'29.46"'s 79³6'36.92"O, 84 m.s.n.m.,.7-IV2013, L. Alarcón, G. Torres, C. Rojas \& B. Esquerre, PRG 14516. Cajamarca: Chota. Macuaco, C. Abad \& L. Vásquez, PRG 6900. Borde de carretera en la comunidad Macuaco - Catache, aprox. 6037'18.88"S 79²17'59.51"'O, 300 m.s.n.m., 27-VIII-2013, L. Alarcón, G. Torres \& B. Esquerre, PRG 14530. Borde de carretera pasando Maichil e Izco, aprox. 6029'44.62"S 7908'39.98”'O, 1335 m.s.n.m. Chota, 23- VI-2013, C. Rojas, B. Esquerre \& R. Cavero, PRG 14521. Bordes de carretera, Potrerillo, 1335 m.s.n.m, 23-VI-2013, C. Rojas, B. Esquerre \& R. Cavero, PRG 14735.

14. Ipomoea quamoclit L., Sp. P1. 1: 159-160 (1753)

= Convolvulus pennatus Desr., Encyl. 3(2): 567-568 (1789)

= Convolvulus pennatifolius Salisb., Prodr. Stirp. Chap. Allerton 124 (1796)

=Convolvulus quamoclit (L.) Spreng., Syst.

Veg. 1: 591 (1825)

Ind. loc.: "Habitat in India"

Lectótipo designado por Biju in Taxon 51: 755 (2002): BM.

Etimología: Nombre introducido por Tournefort (1694) en su obra Élémens de botanique [Tome I] donde claramente hace referencia a la forma de la flor.

Nombre vernáculo local: "Cundeamor", "Quamoclit", "Yedra colorada"

Distribución: Pantropical. EE.UU., ME, DO, HA, JA, NI, CR, PN, CO, VE, SUR, GUF, EC, BR, BO, AR y PE (CU, JU, LO, SM, CA).

Rango altitudinal: 20 a $500 \mathrm{msnm}$.

Hábitat: Bosques secos y comunidades de Cactáceas

Usos: Ornamental

Propagación: Semillas

Conservación: Se encuentra en el Área de Conservación Privada Chaparrí y Refugio de Vida Silvestre Bosques Nublados de Udima.
Fenología: Junio a noviembre.

Observaciones: Ipomoea quamoclit es la única especie del género que presenta hojas laciniadas, y la única del Sección Mina que no presenta aristas carnosas en sus sépalos.

Material estudiado Nuevo reporte para CA Cajamarca: Chota. Borde de carretera en Campamento Carrizal, pasando el Centro Poblado San Carlos - Carhuaquero, aprox. 6035'08.20"'S 79¹4'22.52'O, 500 m.s.n.m, 23-VI-2013, C. Rojas, B. Esquerre \& R. Cavero, PRG 14520. Cerco de jardines, Centro Poblado San Carlos - Carhuaquero, 500 m.s.n.m., 23-VI-2013, C. Rojas, B. Esquerre \& R. Cavero, PRG 14738.

Sección Tricolores McDonald ex D.F.Austin, J. Torrey Bot. Soc. 124(2): 159 (1997)

Tipo: Ipomoea tricolor Cav., Icon. 3(1): 5, pl. 208 (1794[1795])

15. Ipomoea parasitica (Kunth) G. Don, Gen. Hist. 4: 275 (1838)

$\equiv$ Convolvulus parasiticus Kunth, Nov. Gen. Sp. (quarto ed.) 3: 103 (1818)[basión.] = Convolvulus circinnatus Willd. ex Roem. \& Schult., Syst. Veg. 4: 302 (1819)

= Ipomoea perlonga B.L. Rob.in Proc. Amer. Acad. Arts 29: 319- 320 (1894)

Ind. loc.: "Crescit prope Caracas"

Holótipo: P.

Etimología: Del latín "parasiticus”, y del griego "parasitikos" que significa "parásito: el animal o la planta que vive en otros", quizás refiriéndose a la manera de trepar sobre otras especies causándoles estrangulamiento.

Nombre vernáculo local: "campanilla", "quiebra-cajete".

Distribución: ME, GU, NI, CR, VE y PE (CA, LA y PI).

Rango altitudinal: 500 a 1895 msnm

Hábitat: Bosques secos y comunidades de Cactáceas y Pluvisilvas andinas (Galán de Mera et al., 2015).

Usos: Ornamental, en la formación de cercos vivos y también es considerada maleza Propagación: Semillas 
Conservación: Se encuentra en el Área de Conservación Regional Comunal Bosque Moyán-Palacios.

Fenología: Junio a febrero.

Material estudiado Nuevo reporte para LA y CA

Lambayeque: Lambayeque. Bordes de carretera, $\mathrm{Km}$ 17, ruta La Beatita - limón de Porculla, aprox. 5'56'06.22's 79³5'32.70"O, 536 m.s.n.m., 13-IV-2013, G. Torres, L. Alarcón \& B. Esquerre, PRG 14740. Borde de carretera en Kerguer, aprox. $6^{\circ} 11$ '16.33"'S 79³0'42.54"O, 1350 m.s.n.m., 19-VII-2013, G. Torres, L. Alarcón \& B. Esquerre, PRG 14734. Borde de camino en Penachí, aprox. 6 ${ }^{\circ} 10^{\prime} 06.68$ 's 79²8'25.29”'O, 1860 m.s.n.m., 19-VII-2013, G. Torres, L. Alarcón \& B. Esquerre, PRG 14733. Cajamarca: Chota. Pasando Maychil e Izco, aprox. 6²9'19.96"S 7908'04.38'O, 1438 m.s.n.m., 23-VI-2013, C. Rojas, B. Esquerre \& R. Cavero, PRG 14523. Bordes de carretera, pasando Potrerillo, aprox. 629'22.50”'S 79॰08'07.68”O, 1330 m.s.n.m., 23-VI-2013, C. Rojas, B. Esquerre \& R. Cavero, PRG 14732.

16. Ipomoea aristolochiifolia G. Don, Gen. Hist. 4: 277 (1838)

$\equiv$ Convolvulus aristolochiifolius Kunth, Nov. Gen. Sp. (quarto ed.) 3: 101(1818), non Mill., Gard. Dic. (ed. 8): 9 (1768)

= Ipomoea peninsularis Brandegee, Zoë

5: 168(1903)

= Ipomoea tuerckheimii Vatke ex Donn. Sm., Bot. Gaz. 40(1): 8 (1905)

= Ipomoea concinna House, Muhlenbergia 3(3): 42 t. 2, f. c (1907)

= Ipomoea austin-smithii Standl., Publ. Field Mus. Nat. Hist., Bot. Ser. 18(4): 566 (1938)

= Ipomoea cordata L.B. Sm. \& B.G. Schub., Contr. Gray Herb. 127: 31-32, t. 2, f. 33, 34 (1939).

Ind. loc.: "Venezuela"

Holótipo: $\mathrm{P}$

Etimología: Del latín "aristolochiales" de la Fam. Aristolochiaceae, que proviene de Aristolochia: nombre genérico que deriva de las

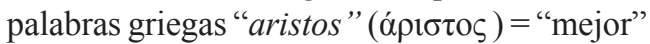

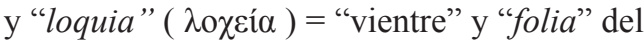

latín "folium" que significa hoja, refiriéndose a la forma de las hojas.

Nombre vernáculo local: "campanilla", "quiebra cajete"

Distribución: ME, GU, NI, CR, CO, VE, EC, BR, BO y PE (AN, AY, JU, PA, CA, PI, LA).

Rango altitudinal: 500 a $1500 \mathrm{msnm}$.

Hábitat: Bosques secos y comunidades de Cactáceas

Usos: Ornamental y también es considerada maleza

Propagación: Semillas

Conservación: Se encuentra en el Área de Conservación Regional Comunal Bosque Moyán-Palacios y Área de Conservación Privada Chaparrí.

Fenología: Junio a febrero.

Material estudiado Nuevo reporte para LA, CA y PI

Lambayeque: Lambayeque. Olmos-Km 25, L. Vásquez, J. Laos, G. Delgado \& E. Sánchez, PRG 4216. Olmos-Km 20, L. Vásquez, J. Laos \& S. Llatas, PRG 4936. Chiclayo. Espinal, C. Abad, PRG 6761. Borde de carretera en Kerguer, aprox. 6 ${ }^{\circ} 11^{\prime} 16.09^{\prime \prime S} 79^{\circ} 30^{\prime} 40.88^{\prime \prime O}, 1356$ m.s.n.m., 19-VII-2013, L. Alarcón, G. Torres \& B. Esquerre, PRG 14741. Cajamarca: Chota. Pasando Potrerillo. aprox. 6 $29^{\circ} 48.05^{\prime}$ 'S $79^{\circ} 08^{\prime} 46.16$ "O, 1333 m.s.n.m., 23-VI-2013, C. Rojas, B. Esquerre \& R. Cavero, 14524 PRG. Bordes de carretera, pasando Potrerillo, 1335 m.s.n.m., 23-VI-2013, C. Rojas, B. Esquerre \& R. Cavero, PRG 14731. Piura: Huancabamba. Quebrada cerca a borde de carretera, Km 29 - 30, camino a Porculla, aprox. 5'53'23.79'"s 79³1'44.19"O, 1250 m.s.n.m., 13-IV-2013, L. Alarcón, G. Torres \& B. Esquerre, PRG 14743.

Sección Pedatisectae House (McDonald)

17. Ipomoea cairica (L.) Sweet, Hort. Brit. 2872871827.

= Convolvulus cairicus L., Syst. Nat. (ed. 10) 2: 922 (1759)

= Ipomoea senegalensi Lam., Tabl. Encycl. 1: 464 (1791)

= Ipomoea pentaphylla Cav., Icon. 3: 29 (1794) 
Ind. loc.: no especifica

Lectótipo designado por Bosser \& Heine in Bosser \& al. (ed.), Fl. Mascareignes 127: 32 (2000).

Etimología: Del adjetivo latino "cairicus, $a, u m "=$ de El Cairo.

Nombre vernáculo local: "enredadera"

Distribución: EE.UU., JA, CO, VE, GU, EC, BR, BO, PAR y PE (AR, LL, CA, LA).

Rango altitudinal: 0 a $1200 \mathrm{msnm}$.

Hábitat: Bosques secos y comunidades de Cactáceas

Usos: Ornamental, en la formación de cercos vivos y también es considerada maleza

Propagación: Semillas

Conservación: Se encuentra en el Área de Conservación Privada Chaparrí y Refugio de Vida Silvestre Bosques Nublados de Udima.

Fenología: Agosto a febrero.

Material estudiado Nuevo reporte para LA Lambayeque: Chiclayo. Fundo la Joyita, Pimentel, aprox. 6048'12.39"'s 7953'03.55"O, 20 m.s.n.m., 03-IV-2013, L. Alarcón \& G. Torres, PRG 14513.

18. Ipomoea wrightii A. Gray, Syn. Fl. N. Amer. 2(1): 213 (1878)

= Convolvulus heptaphyllus Rottler \& Willd., Ges. Naturf. Freunde Berlin Neue Schriften 4: 196 (1803).

= Ipomoea pulchella Roth, Nov. Pl. Sp.115 (1821)

= Convolvulus heptaphyllus Roxb., Fl. Ind., ed. 1820 2: 66 (1824)

Ind. loc.: "USA: Texas"

Holótipo: GH

Etimología: Esta especie fue dedicada a Charles Wright, botánico norteamericano que estudió plantas en Cuba en el siglo XIX.

Nombre vernáculo local: "don diego con hojas de palmera"

Distribución: América tropical. EE.UU., DO, HA, JA, NI, VE, EC, BR, AR, PAR y PE (LA, LO).

Rango altitudinal: 0 a $500 \mathrm{msnm}$
Hábitat: Bosques secos y comunidades de Cactáceas

Usos: Es considerada maleza en cultivos de arroz, caña de azúcar y algodón

Propagación: Semillas

Conservación: Se encuentra en el Santuario Histórico Bosque de Pomac

Fenología: Noviembre a abril.

Material estudiado Reportada para LA (Brako \& Zarucchi, 1993)

Lambayeque: Lambayeque. Santa María Lambayeque, C. Cueva, S. Llatas \& C. Estela, PRG 6341. Yencala-Lambayeque, C. Cueva, S, Llatas \& C. Estela, PRG 6398. El Cienago, C. Abad \& J. Orrillo, PRG 6781. Ferreñafe. Chisco blanco, A. Díaz \& L. Cerna, PRG 431. Papayo-Pitipo, A. Díaz \& S. Eche, PRG 1846. Pítipo, S. Llatas, HUT 16744. Chiclayo. Entre Chiclayo y Ferreñafe, L. Vasquez \& H. Ocampo, PRG 1482. Reque, J. Laos \& S, Llatas, PRG 5395. Bordes de cultivos de caña de azúcar en Pucalá, aprox. 6०46'32.27'S 79³6'34.57'O, 84 m.s.n.m., 07-IV-2013, L. Alarcón, G. Torres, C. Rojas \& B. Esquerre, PRG 14517.

Gen. Merremia Dennst. ex. Endl., Gen. Pl. 18: 1403 (1841)

Tipo: Merremia hederacea (Burm. f.) Hallier f., Bot. Jahrb. Syst. 18(1-2): 118 (1893): $\mathrm{G}$

Etimologia: En honor a Blasius Merrem (1761-1824), botánico, matemático y físico alemán.

Clave de especies de Merremia para la región Lambayeque y zonas adyacentes

1. Hojas simples; flores amarillas M. umbellata

- Hojas 5 palmatipartidas; flores blancas o de color crema

2. Peciolo hirsuto de 2,5-11 cm de largo; sépalos externos de mayor tamaño que los internos ..................................... M. aegyptia

- Peciolo glabro de 0,5-5,5 cm de largo; sepalos internos de mayor tamaño que los externos

M. quinquefolia

19. Merremia aegyptia (L.) Urb., Symb. Antill. 4: 505 (1910) =Ipomoea aegyptia L., Sp. Pl. 1: 162 (1753) 
=Ipomoea pilosa Cav., Icon. 4: 11 t. 323

(1797)

= Convolvulus munitus Wall., Numer. List 1354 (1829)

Ind. loc.: "USA: Texas"

Lectótipo designado por D.F.Austin in Fl. Ecuador 16: 84 (1982): LINN

Etimología: “aegyptia”: epíteto geográfico que alude a su localización en Egipto.

Nombre vernáculo local: "campanilla", "gloria de la mañana peluda"

Distribución: Pantropical, ME, GU, NI, $\mathrm{CR}, \mathrm{CU}, \mathrm{PR}, \mathrm{CO}, \mathrm{EC}, \mathrm{BR}$ y $\mathrm{PE}$ (AM, CU, JU, LA).

Rango Altitudinal: 0 a $500 \mathrm{msnm}$.

Hábitat: Bosques secos y comunidades de Cactáceas

Usos: Considerada maleza

Propagación: Semillas

Conservación: Se encuentra en el Área de Conservación Regional Comunal Bosque Moyán-Palacios, Santuario Histórico Bosque de Pómac y Área de Conservación Privada Chaparrí.

Fenología: Octubre a abril

Material estudiado Nuevo reporte para LA Lambayeque: Lambayeque. Olmos, Portachuelo, C. Fiestas, PRG 1341. Olmos, A. Díaz \& L. Vásquez, PRG 273. Olmos, A. Díaz, PRG 293. Olmos-Km 890, A. Díaz, PRG 1917. Motupe, R. Ferreyra, USM 5878. Motupe, R. Ferreyra, USM 9057. Motupe, Vivero, A. Díaz \& S.M. Callacna, PRG 2766. Motupe, A. Díaz, PRG 2872. Motupe, Quebrada del Tabacal, C. Abad \& S. Llatas, PRG 6752. Jayanca, A. López, HUT 2455. Chiclayo: Chongoyape, Tinajones, A. Díaz \& L. Cerna, PRG 375. Chongoyape, A. Díaz, PRG 1290; Chongoyape, A. Díaz \& L. Cerna, PRG 1278. Chongoyape, C. Abad \& S. Llatas, PRG 6755. Reque, S. Llatas, PRG 5897. Mocupe, L. Vásquez \& H. Ocampo, PRG 1455.

20. Merremia quinquefolia (L.) Hallier f., Bot. Jahrb. Syst. 16: 552 (1893)

三Ipomoea quinquefolia L., Sp. Pl. 1: 162 (1753)

= Convolvulus quinquefolius (L.) L., Syst.
Nat. (ed. 10) 923 (1759)

= Convolvulus hispaniolae Spreng. Syst.

Veg. 1: 590 (1825)

= Convolvulus ampelopsifolius Cham. \&

Schltdl., Linnaea 5: 118 (1830)

Ind. loc.: "Habitat in America"

Lectótipo designado por D.F.Austin in Woodson \& Schery (ed.), Ann. Missouri Bot. Gard. 62: 182 (1975).

Etimología: "Quinquefolius, -a, -um."lat. "quinquefolius, -a, -um" = que tiene cinco hojas. Se refiere a las hojas palmaticompuestas de 5 foliolos.

Nombre vernáculo local: "cinco hojas",

Distribución: EE.UU., ME, JA, GU, NI, CR, CU, PR, las Antillas, CO, EC, BR y PE (AM, AN, HU, LA, JU).

Rango altitudinal: 0 a $500 \mathrm{msnm}$.

Hábitat: Bosques secos y comunidades de Cactáceas

Usos: Por formar matas densas se utiliza para cercos de terrenos de cultivo

Propagación: Semillas y esquejes

Conservación: Se encuentra en el Santuario Histórico Bosque de Pómac y Área de Conservación Privada Chaparrí.

Fenología: Noviembre a julio.

Material estudiado Nuevo reporte para LA Lambayeque: Lambayeque. Motupe, Huerto frutícola, A. Díaz, PRG 4062. Mochumí, A. Díaz \& L. Cerna, PRG 4478. Ferreñafe. Borde de camino a Santa Clara, antes del Puente Colgante Pítipo, aprox. $6^{\circ} 26.841^{\prime}$ 'S $79^{\circ} 44.001^{\prime} \mathrm{O}, 84$ m.s.n.m., 13-VII-2013, L. Alarcón, G. Torres \& B. Esquerre, PRG 14526. Chiclayo. Oyotún, J. Laos, PRG 6737. Reque, S. Llatas, PRG 6073. Luya, A. Díaz \& L. Cerna, PRG 417. Zaña, C. Abad, J. Escurra, E. Benavides \& S. Llatas, PRG 6770. Chongoyape, C. Abad, PRG 6745. Chongoyape, C. Abad, J. Laos \& S. Llatas, PRG 6754. Fundo la Joyita, Pimentel, aprox. 6 48'26.72"S 7953'03.22"O, 19 m.s.n.m., 03-IV- 2013, L. Alarcón \& G. Torres, PRG 14511.

21. Merremia umbellata (L.) Hallier f. in Bot. Jahrb. Syst. 16(4-5): 552(1893)

三Convolvulus umbellatus L., Sp. P1. 1: 155 (1753) 
= Convolvulus aristolochiifolius Mill. Gard. Dict. (ed. 8): 9 (1768), non Kunth, Nov. Gen. Sp. (ed. 4) 3: 102 (1818[1819]).

= Convolvulus caracassanus Willd. ex Roem. \& Schult., Syst. Veg. 4: 301 (1819) = Convolvulus cymosus Desr., Encycl. 3: 556 (1789)

Ind. loc.: "Habitat in Martinica, Domingo, Jamaica"

Lectótipo designado por D.F.Austin in Florida Sci. 42 : 221 (1979)

Etimología: epíteto "Umbellatus, -a, -um" -bot. umbellatus, - $a$, -um = umbelado, provisto de umbelas Se refiere a la forma de la inflorescencia.

Nombre vernáculo local: "amole de venado", "bejuco manzo", "campanilla", "cuajo de hule", "cuelga-tabaco" y "jícama cimarrona".

Distribución: Pantropical, ME, GU, NI, CR, CU, PR, CO, EC, BR y PE (AM, HU, JU, LO, MD, PI, CA, LA, SM).

Rango altitudinal: 0 a $7000 \mathrm{msnm}$

Hábitat: Bosques secos y comunidades de Cactáceas

Usos: Maleza

Propagación: Semillas

Conservación: Se encuentra en el Santuario Histórico Bosque de Pómac

Fenología: Noviembre a julio.

Material estudiado Nuevo reporte para LA Lambayeque: Lambayeque. Lambayeque, L. Vásquez \& H. Ocampo, PRG 1444. Íllimo, San Juan, C. Abad, PRG 6746. Chiclayo: Oyotún, La Jara, J. Laos \& H. Aurazo, PRG 5636. Reque, J. Laos \& S. Llatas, PRG 6300. Reque, C. Abad \& S. Llatas, PRG 6767. Entre Zaña y Cayaltí, A. López, HUT 1086.

AGRADECIMIENTOS. A Guillermo DelgadoParedes, por las facilidades en el uso del Laboratorio de Cultivo de Tejidos Vegetales y Recursos Genéticos. A la memoria del Prof. Dr. Santos R. Llatas-Quiroz por sus enseñanzas y facilidades para realizar el trabajo de campo. A Consuelo Rojas-Idrogo por sus orientaciones en el trabajo de campo. A Boris Esquerre, Ruth Cavero y Yuriko Murillo, por su compañía, apoyo y amistad durante las campañas botánicas.

\section{BIBLIOGRAFÍA}

APG IV -2016- An update of the Angiosperm Phylogeny Group classification for the orders and families of flowering plants: APG III. Bot. J. Linn. Soc. 181:1-20.

ABAD, C -1983- Convolvuláceas silvestres del departamento de Lambayeque. Tesis para Licenciatura. Facultad de Ciencias Biológicas. Universidad Nacional Pedro Ruiz Gallo. Lambayeque - Perú.

AUSTIN, D.F -1975a- Convolvulaceae. Flora of Panama. Ann. Missouri Bot. Gard. 62:157-224.

AUSTIN, D.F -1975b- Typification of the New World subdivisions of Ipomoea L. (Convolvulaceae). Taxon 24:107-110.

AUSTIN, D.F -1979- An infrageneric classification for Ipomoea (Convolvulaceae). Taxon 28: 359-361.

AUSTIN, D.F. \& Z. HUAMÁN -1996- A sinopsis of Ipomoea (Convolvulaceae) in the Americas. Taxon 29:501-502.

AUSTIN, D. F -1997- Convolvulaceae morning glory family. Journal of the Arizona-Nevada Academy of Science. Departament of Biological Sciences, Florida Atlantic University. U.S.A.

BRAKO, L. \& J.L. ZARUCCHI -1993- Catalogue of the flowering plants and gymnosperms of Perú/Catálogo de las Angiospermas y Gimnospermas del Perú. Monogr. Syst. Bot. 45: 365-374.

CHIARINI, F. \& L. ARIZA -2006- Flora Fanerogámica Argentina: Convolvulaceae. Instituto Multidisciplinario de Biología Vegetal: Universidad Nacional de Córdoba. CONICET, Córdoba.

FELGER, R., D.F. AUSTIN, T. VAN DEVENDER, J. SANCHEZ-ESCALANTE \& M. COSTEA -2012- Convolvulaceae: Convolvulus, Cressa, Dichondra, Evolvulus, Ipomoea, Jacquemontia, Merremia and Operculina of Sonora, México. J. Bot. Res. Inst. Tex. 6: 459-527.

GALÁN DE MERA, A., I. SÁNCHEZ VEGA, J. MONTOYA QUINO, E. LINARES PEREA, 
J. CAMPOS DE LA CRUZ \& J.A. VICENTE ORELLANA -2015- La vegetación del Norte del Perú: De los bosques a la jalca en Cajamarca. Acta Bot. Malacitana 40: 157-190.

LEÓN, B -2006- Convolvulaceae endémicas del Perú. El libro rojo de las plantas endémicas del Perú. Rev. Perú. Biol. 13(2): 266s-267s.

LLATAS, S -2011- Diversidad Taxonómica de la Flora Fanerogámica de la Región Lambayeque (1976-2010). Tesis doctoral. Facultad de Ciencias Biológicas. Universidad Nacional Pedro Ruiz Gallo. Lambayeque - Perú.

LLATAS, S. \& M. LÓPEZ MENESES -2005Bosques montanos relictos en Kañaris (Lambayeque, Perú). Rev. Per. Biol. 12:299-308.

LLATAS, S., J.A. VICENTE ORELLANA \& A. GALÁN DE MERA -1997- Proyecto de una flora del Departamento de Lambayeque (Perú). Anales Jard. Bot. Madrid 55: 176-181.

MOSTACERO, J., F. MEJÍA \& O. GAMARRA -2002- Taxonomía de las Fanerógamas Útiles del Perú. Edit. Normas Legales S.a.C. CONCYTEC. Vol. II, pp. 702-719.

NAMANDA, S., R. GATIMU, S. AGILI, S. KHISA, I. NDYETABULA \& C. BAGAMBISA -2015Micropropagation and hardening sweetpotato tissue culture plantlets. A manual developed from the SASHA Project's experience in Tazania.
International Potato Center, Nairobi, Kenya. $100 \mathrm{p}$.

NASKAR, K.R. -1990- Aquatic \& semi aquatic plants of lower Ganga delta. Daya Publishing House, Delhi, India. Pp. 173-174.

RIVAS-MARTÍNEZ, S., G. NAVARRO, A. PENAS \& M. COSTA -2011- Biogeographic Map of South America. A preliminary survey. Int. J. Geobot. Res. 1: 21-40+Map.

SHINNERS, Ll. -1965- "Untypification for Ipomoea nil"

THE FIELD MUSEUM NEOTROPICAL HERBARIUM SPECIMENS -2014- (en línea). [Fecha de consulta: 11 mayo 2016]. Disponible en: http://fm1.fieldmuseum.org/vrrc.

THE PLANT LIST (en línea). [en línea]. Version 1.1, publicado en Setiembre del 2013. [última fecha de consulta: 11 mayo 2016]. Disponible en: http://www.theplantlist.org/

ULLOA ULLOA, C., J.L. ZARUCCHI \& B. LEÓN -2004- Diez años de adiciones a la flora del Perú: 1993-2003. Arnaldoa (Ed. Especial): 1-242.

WAGNER, H -1973- The chemistry of the resin glycosides of the Convolvulaceae family, in: Bendz G., Santesson J. (eds.). Medicine and Natural Sciences. Chemistry in Botanical Classification, Academic Press. New York. p. 235-240. 